\title{
Represión, control y disciplinamiento en la Universidad de Buenos Aires durante la última dictadura (1976-1983)
}

\section{Resumen}

En los últimos años el campo de estudios sobre la universidad argentina y el movimiento estudiantil se han consolidado. Sin embargo, el sector estudiantil universitario durante la última dictadura ha permanecido poco analizado. Este trabajo se propone completar dicha vacancia, focalizando sobre las experiencias de dicho actor durante los años de represión más feroz. En particular, nos proponemos analizar el desarrollo de la política represiva y de disciplinamiento en la Universidad de Buenos Aires (UBA), entre 1976 y 1983, y sus efectos en la vida estudiantil, trabajando tanto con fuentes documentales como con testimonios orales. Así, complejizaremos nuestra comprensión sobre la vida universitaria más allá de la normativa aprobada.

Palabras clave: Represión, Disciplinamiento, Universidad de Buenos Aires, Estudiantes.

\section{Abstract}

In the last years the field of studies on the Argentine university and the student movement has consolidated. However, the sector of university students during the last dictatorship has remained poorly analyzed. This work aims to contribute to completing this vacancy, focusing on the experiences of this actor during the years of repression. In particular, we intend to analyze the development of repressive and disciplinary policies at the University of Buenos Aires (UBA) between 1976 and 1983, and its effects on student life. For this, we work with both documentary sources and oral testimonies. In this way, we seek to contribute to building a more complex knowledge of university life.

Key words: Repression, Disciplining, University of Buenos Aires, Students.

POR GUADALUPE A. SEIA. Doctora en Ciencias Sociales (Universidad de Buenos Aires) Magister en Historia Contemporánea (Universidad Nacional de General Sarmiento) y Licenciada en Sociología (Universidad de Buenos Aires). Se desempeña como becaria posdoctoral de CONICET, con lugar de trabajo en el Instituto de Altos Estudios Sociales, Universidad Nacional de San Martín (IDAES-UNSAM). guadalupeseia@gmail.com 


\section{Introducción}

En el presente artículo analizaremos la política represiva y de disciplinamento hacia el estudiantado desarrollada en la Universidad de Buenos Aires durante la última dictadura en Argentina (1976-1983). En particular, consideraremos las medidas tomadas por las autoridades de la universidad porteña, así como también los efectos en la vida de los estudiantes, considerando sus experiencias en dicho contexto. ${ }^{1}$

El campo de estudios sobre la universidad argentina como objeto de estudio en la historia reciente (1966-1983) se ha fortalecido en los últimos años (Kaufmann, 2001, 2003; Rodríguez, 2014; Friedemann, 2015; Águila,2014; Algañaraz Soria,2014; Vera de Flachs, 2015; Gil y Díaz, 2014; Orbe, 2014; Seia, 2016a; Mendonça, 2016, etc.). Laura Rodríguez (2015) ha realizado un esfuerzo por caracterizar en términos globales la política universitaria de la última dictadura. En este texto analizaremos la implementación de los aspectos represivos y de disciplinamiento de dicha política en la principal universidad pública nacional.

Asimismo, los análisis sobre la movilización y organización estudiantil (Millán, 2015; Califa, 2015; Pis Diez, 2016; Bonavena, 2016; etc.) consolidaron un área de estudios. Sin embargo, el sector estudiantil universitario durante la última dictadura ha permanecido poco estudiado (Seia 2016a; Perelet al, 2003; Luciani, 2017). Al respecto, los tempranos trabajos de Laura Polak y Juan Carlos Gorbier (1986) y Fernando Pedrosa (2002) han descripto de modo general el silenciamiento y temor instalados en los claustros que hasta no hacía mucho se habían destacado por la efervescencia social y la movilización estudiantil. Pedrosa sostiene que el estudiantado se vio sometido a una rigurosa vigilancia y enorme presión para adaptarse a un modelo de estudiante "apolítico" que incluía rasgos como la obediencia acrítica y complaciente con la realidad universitaria y nacional. Los estudiantes aprendieron a vivir bajo esa atmosfera, en donde el límite entre lo permitido y lo prohibido era difuso. Así, se habría ido articulando entre los estudiantes una especie de "sentido común difuso y tácito" sobre las cosas que "no se hacían" y "no se discutían". En este trabajo nos ocuparemos de dichos "aprendizajes" que influenciaron las prácticas de los y las jóvenes que pasaron por los claustros porte- 
ños entre 1976 y 1983. Abordaremos sus experiencias para reconstruir las vivencias a lo largo de la etapa, procurando responder a ciertos interrogantes: ¿cómo respondieron las y los estudiantes a la represión y la vigilancia? ¿Qué efectos tuvo dicha política en la vida estudiantil porteña?

Hemos trabajado con fuentes documentales y con testimonios orales recolectados en el marco de nuestra investigación. Apostamos a complejizar nuestra comprensión sobre la vida universitaria porteña durante estos años más allá de la normativa aprobada por el Ministerio de Cultura y Educación (MCE) y el rectorado.

\section{La universidad bajo la última dictadura}

El golpe de Estado del 24 de marzo de 1976 instaló en Argentina el "Estado Terrorista" (Duhalde, 2013), caracterizado por la militarización del aparato del estado y un alto contenido represivo que pretendió la aniquilación física de sus opositores y el amedrentamiento de la población a través del terror. El dispositivo represivo incluyó el secuestro, la tortura, la desaparición sistemática de personas y el funcionamiento de centros clandestinos de detención. Continuando y profundizando lo iniciado por el gobierno peronista (Friedemann, 2015; Califa y Millán, 2016; Franco, 2012), la universidad fue uno de los terrenos donde la represión paraestatal y estatal se asentó. Al finalizar la etapa, más del 20 por ciento de los desaparecidos fueron estudiantes (CONADEP). Las cifras disponibles sobre las víctimas de la represión en la UBA indican que hubo 636 desapariciones (Rodríguez, 2015). ${ }^{2}$

Para las autoridades de la dictadura, la universidad recibida era la "universidad del caos" y "de la violencia", el ámbito por excelencia de penetración de la "subversión”. Para las Fuerzas Armadas (FFAA en adelante) y el MCE, el problema "más grave" de la educación argentina se localizaba en la universidad debido a sus características particulares, la edad del estudiantado y su trascendencia política. Allí la "subversión" accionaba con un sentido prioritario (MCE, 1977). Las organizaciones estudiantiles fueron identificadas como “enemigas activas" y perseguidas sistemáticamente.

El origen de esta situación fue identificado por las autoridades del autodenominado "Proceso de
Reorganización Nacional” (“PRN") en la Reforma Universitaria de 1918 que había sido influenciada por la Revolución Bolchevique de 1917 (MCE, 1977). A partir de entonces los estudiantes habían comenzado a movilizarse y politizarse, pensando que incluso "debían" tener injerencia en el gobierno de las casas de estudios superiores. En 1973, regresaron a los claustros los sectores izquierdistas desplazados por el gobierno de Onganía en 1966, produciendo "la destrucción más intensa que se conoce de la Universidad, y el desarrollo del proceso de captación de jóvenes para integrar los cuadros del terrorismo..." (FFAA, 1979).

\section{El establecimiento de la "paz", el disciplinamiento juvenil y la erradicación de la política y la organización estudiantil eran condiciones para el funcionamiento de la universidad.}

Las Universidades Nacionales (UUNN) fueron intervenidas y puestas a disposición del Poder Ejecutivo Nacional (PEN, 1976a y b). Se procedió a restaurar "el orden imprescindible" mediante la acción de los delegados de las FFAA y la colaboración de "civiles con alto prestigio académico". En la UBA, el delegado militar Capitán de Navío Edmundo Said procedió a "cesar en funciones" al personal docente y no docente de la UBA. Más de 150 investigadores y profesores fueron separados de sus cargos (Res. N $71-77 / 1976$; etc.). En paralelo, se incorporó a cientos de docentes que habían sido suspendidos en 1973 (Res. $N^{\circ} 14,1,144 / 1976$; etc.).

Para los estudiantes se volvió norma la exigencia para la inscripción de un certificado de domicilio actualizado expedido por las autoridades correspondientes (Policía Federal) y un certificado de buena conducta o de antecedentes penales, junto al certificado de estudios secundarios y la aprobación de los exámenes de ingreso (Seia, 2016a). Esto se combinó con la presencia constante de las fuerzas represivas en las instalaciones, 
mientras que la presencia activa de bandas paramilitares al interior de los claustros mermó. Asimismo, el delegado militar de la UBA aprobó el régimen de disciplina al que estarían sometidos todos los estudiantes de la universidad. Los "actos de indisciplina" sancionados incluían: desobediencia ante las órdenes de docentes y autoridades; falta de respeto o agresión hacia las mismas; participación en desordenes en el ámbito universitario; actitudes o expresiones "contrarias al decoro o a las buenas costumbres"; instigación a "desmanes, tumultos u ocupaciones de locales universitarios"; actividades que asumieran "formas de adoctrinamiento, propaganda, proselitismo o agitación de carácter político y gremial, docente, estudiantil y no docente", que denotara "peligrosidad para la seguridad nacional”, etc. (Res. S/No/1976).

Said ocupó el cargo de delegado militar en la UBA hasta el 6 de agosto de 1976. Desde ese momento hasta fines de 1983, las máximas autoridades fueron actores civiles que poseían una trayectoria en el sistema de educación superior y en dicha casa de estudios, varios se habían desempeñado como decanos durante la intervención de la dictadura anterior (Seia, 2014; Rodríguez, 2015). ${ }^{3}$ A pesar de ciertas diferencias que mantuvieron algunos rectores con el MCE (Seia, 2016c) es necesario destacar que todos estos actores acordaban en la necesidad de "ordenar los claustros", línea de continuidad con la intervención militar. El establecimiento de la "paz", el disciplinamiento juvenil y la erradicación de la política y la organización estudiantil eran condiciones para el funcionamiento de la universidad. Así, la política de "depuración" a través de las bajas de docentes no se interrumpió. También continuó vigente la disposición de que todos los viajes por parte de docentes de esta universidad debían ser aprobados por el rector, la presencia de las fuerzas represivas, y la exigencia de certificado de domicilio y de buena conducta a los estudiantes (Res. N³77/1976).

Según los testimonios analizados, los controles en los ingresos de las unidades académicas por parte de personal uniformado "armados con fusiles enormes" no se evitaron hasta el final de la etapa. Prácticamente la totalidad de los entrevistados describen las requisas (abrían y vaciaban sus bolsos y carteras, revisaban el interior de los libros "para ver si se caía algo", buscaban papeles, etc.) y la exigencia de la libreta para ser autori- 
"boca en boca" por lo bajo también posibilitó que los jóvenes mantuvieran cierta comunicación e información sobre la realidad.

Quienes cursaron su carrera exclusivamente durante la etapa dictatorial reconstruyen una línea de mayor a menor intensidad en la represión en las facultades. Los testimonios localizan los años "más duros" de la represión entre 1976 y 1978. Según sostienen, los controles se fueron relajando en 1979 ante la presión internacional por la violación de los derechos humanos y con la llegada de Roberto Eduardo Viola, y en 1982 con la guerra en Malvinas: "A esa altura, años 80, 81, sabíamos que había un riesgo. Pero también entendíamos que la represión más fuerte había pasado" (A. Romano, 2013). También quienes ingresaron a partir de 1980 y más específicamente en 1982, relatan una vigilancia y control, pero más laxa que sus compañeros mayores. Pablo Alabarces (Letras) cuenta que él experimentaba las condiciones de seguridad que imponían los militantes "más viejos" como exagerado. Eduardo (Historia) relata que como ingresó un mes antes del inicio de la Guerra de Malvinas casi no vivió un clima represivo en la facultad.

Entendemos que estas experiencias deben ser complejizadas considerando a cada facultad. Según quienes estudiaron Ciencias Exactas, ubicada en "Ciudad Universitaria" en una zona aislada del resto de las unidades académicas, la vigilancia y el control se mantuvieron estrictos prácticamente hasta el final de la dictadura. Carolina Vera y Juan Pablo Paz, a pesar de sus diferencias de cohorte -él entró en 1978 y ella en 1980-, coinciden en que las fuerzas represivas continuaron teniendo una presencia activa. Al respecto contamos con los informes de inteligencia (Fuerza Aérea Argentina, 1982; Consejo de Rectores de Universidades Nacionales, 1982) y las experiencias de detención de Paz en 1981 y Pablo Mauas en 1982. En contraste, en Filosofía y Letras, Ciencias Económicas, Sociología y Derecho, estudiantes de diferentes sub-generaciones coincidieron en que desde 1979 se vivió una primera "relajación" de la vigilancia que se profundizó con la llegada de Viola al gobierno y con la Guerra de Malvinas.

Al respecto de las vivencias de la "relajación" de la represión a lo largo de los años es necesario plantear algunas consideraciones. Debemos destacar que a pesar de que los testimonios sostienen que la intensidad de los controles y la represión fue disminuyendo hacia 1979, diferentes fuentes coinciden en indicar que las fuerzas represivas uniformadas y de civil permanecieron en los claustros hasta el último día del gobierno militar. De este modo, entendemos posible pensar que esas percepciones tuvieron que ver con la disminución de las desapariciones y asesinatos a partir de 1978 a nivel nacional $1^{5}$; la crisis política que atravesó el gobierno dictatorial a partir de la derrota bélica en 1982; pero también con ciertos procesos de naturalización del control en las facultades.

\section{Naturalización, "zonas grises" e invisibilización}

Algunos testimonios, particularmente de Filosofía y Letras y Derecho, recuerdan que como los guardias ya "conocían" a los estudiantes regulares, el control se iba "relajando" por lo menos hacia aquellos jóvenes que no parecían "sospechosos" (V. Cipolla, J. Cernadas, A. Cattaruzza, P. Alabarces, 2015). Pablo Alabarces recuerda que había construido un vínculo "amistoso" con quien custodiaba la entrada de la sede de Marcelo T. de Alvear. A través de esa relación, sostiene, consiguió que la vigilancia sobre su persona fuera menos pesada, dándole margen para actuar políticamente:

...yo le daba charla al "miliquito" que estaba en la puerta, un cana, un "canita"...lo saludaba, un tipo macanudo y un día, mi contacto del PTS, me dice "te tengo que pasar unos volantes", me encuentra en la puerta de la facultad y está sacando el paquete, de golpe se pone blanca, me doy vuelta y veo que está el "canita" mirándonos, me acerco y le digo: "¿Qué te pasa boludo? Me está dando unas cosas", "Ah no disculpa, loco, perdóname”... (P. Alabarces, op. cit.)

Observamos cómo en la reconstrucción de las vivencias cotidianas, los grises aparecen como un elemento sumamente importante a considerar. Tenemos representaciones acerca de cómo los agentes de las fuerzas represivas se volvían parte de "la normalidad" del funcionamiento de esas casas de estudio, que identificaban y conocían a los estudiantes y con quienes los 
jóvenes mantenían una relación más o menos cordial y cotidiana. Así, es posible dar cuenta de las diversas aristas de una relación compleja entre las fuerzas represivas y los estudiantes. En el caso mencionado, el representante de las fuerzas represivas no era visto como una figura de "temer", sino más bien una figura minimizada ("el miliquito"). A la vez, ese trato cordial terminó por ser una estrategia para obtener mayor grado de acción en una facultad sumamente vigilada. De este modo, evitó que lo encontraran con materiales políticos y que incluso le pidiera disculpas. Otros testimonios de estudiantes de la Facultad de Filosofía y Letras coinciden (sin plantear una relación de "amistad" con los guardias) en que al ser pocos estudiantes y los guardias más o menos permanentes se llegaban a identificar de verse día tras día. En efecto, identifican una "relajación" en los controles para ingresar a la facultad por dicha situación. Después de meses o años, se volvía una acción rutinaria tanto para los jóvenes como para el propio guardia.

... existen numerosos casos en que

los estudiantes con participación política

sufrieron los efectos de la vigilancia y de la represión de modo directo.

En Ciencias Naturales, mientras que los testimonios sostienen que la vigilancia no se interrumpió y no destacan una "relajación" de la misma, coinciden en que como estudiantes ellos fueron aprendiendo a manejarse en la situación que les tocaba estudiar. Algunos lo hicieron con temor y para resguardar su seguridad, otros porque era necesario "tener cuidado". Identificamos actitudes de acostumbramiento y naturalización de la presencia de las fuerzas de seguridad, las requisas, la exigencia de documentación para ingresar o incluso tener policías de civil en el aula. Estas situaciones constituyeron la realidad cotidiana de estudio y socialización: 
tenías que presentar la libreta universitaria porque si te la olvidabas, no entrabas. Yo lo tomaba como una molestia, pero era chica y me daba la sensación de que eran cosas que formaban parte de una nueva etapa... (H. Pizarro, 2012)

También es posible identificar en Filosofía, cierto acostumbramiento y la naturalización de la vigilancia. Varios testimonios, aun reconociendo la presencia de la vigilancia, afirmaban no haber tenido "malas experiencias" ni haber presenciado acciones "abusivas" de su parte. Luchas Luchilo y Alejandro Cattaruzza (estudiantes de Historia) sí recordaron algunos "incidentes". En sus relatos estos sucesos aparecen provistos de una escasa trascendencia, "excepcionales" y que no tuvieron un desenlace trágico (muerte o desaparición de estudiantes). Es posible identificar una relativa naturalización de la presencia de las fuerzas represivas que, según comentan, estaban allí pero no ejercían "activamente" la represión contra los estudiantes salvo "excepciones", o bien en comparación con otras situaciones, su presencia no era una "amenaza fuerte". Esto sucede tanto entre aquellos estudiantes que tenían actividad política como quienes no.

En ese sentido, tal como lo destaca Laura Luciani (2017), el "shock" de ver a las fuerzas de seguridad en los pasillos y aulas sería mayor para aquellos que pasaron por la experiencia de cursar y militar en la universidad hasta 1974. En cambio, fue experimentado como algo cotidiano y más "normal" por aquellos actores que no fueron partícipes o testigos del proceso de activación y movilización política estudiantil de los años anteriores. Quienes cursaron toda su carrera en los años de la dictadura, la presencia policial es observable para algunos más notoria, coinciden en que su hostilidad se va diluyendo, relatan que no presenciaron ningún hecho "particularmente" represivo o los que relatan son "minimizados". En ese sentido, la presencia represiva se vuelve parte del marco cotidiano en el que los estudiantes debían actuar, incluso para llegar a ser invisibilizada: "Yo formo parte de una generación que entró en la Facultad cuando ya estaba la dictadura y la mayoría de nosotros éramos bastante ignorantes acerca de las cosas más pesadas que estaban ocurriendo." (A. Romano, 2013).

Al respecto, debemos interrogarnos acerca de las modalidades represivas específicas de la dictadura, en tanto, en los testimonios analizados no aparecen menciones a enfrentamientos, secuestros o asesinatos al interior de los claustros. Respaldando la hipótesis de que, a diferencia de la etapa inmediata anterior, habría habido escasos secuestros o asesinatos en las facultades, encontramos los datos de algunas desapariciones de estudiantes. Inés Olleros fue detenida en 1977 
cuando viajaba en colectivo por tener materiales del Partido Comunista, trasladada a una comisaría y luego desaparecida. Eduardo Corvalán y Antonio Misetich de Ciencias Exactas (Díaz de Guijarro et al., 2015), Hernán Abriata, Hernán Nuguer y Pablo Glarcep de Arquitectura fueron secuestrados en sus domicilios.

En cambio, resultó recurrente que los testimonios destacaran que tenían noción de la "ausencia" de otros estudiantes, compañeros de cursada, aunque afirmaron no haber presenciado su desaparición o bien, no haber terminado de comprender lo que sucedía. A pesar de que hubiera hechos que les llamaran la atención era "mejor" no preguntar, no cuestionar:

si bien no presencié ninguna detención dentro de la facultad, sí recuerdo que, en alguna ocasión, dejaba de venir un compañero y los docentes le pedían a sus amigos que averiguaran qué le pasaba que no concurría y las repuestas eran que la familia no daba información, y todo quedaba ahí, nadie hacía más preguntas ni comentarios. Se sabía, tácitamente, que no había que indagar. (V. Ferraro, 2015)

Tampoco debemos pasar por alto las experiencias de estudiantes que tenían actividad política de algún tipo y fueron víctima de amenazas, detenciones, interrogatorios y algunos golpes. En Filosofía, Nancy fue amenazada en la facultad durante 1974; mientras que ya en dictadura Patricio Geli casi sufre el allanamiento de su casa que fue impedido, según relata, porque un vecino de él era juez; Pablo Alabarces fue interrogado en la "oficina" de la sede de Marcelo T. de Alvear. En Ciencias Exactas, Gabriel Bilmes fue detenido en 1974, Juan Pablo y Pablo Mauas fueron detenidos en la facultad en 1982. En Arquitectura, Andrés Weiger, presidente del Centro de estudiantes en 1974, fue informado que figuraba en una lista de estudiantes a "eliminar". Es decir, existen numerosos casos en que los estudiantes con participación política sufrieron los efectos de la vigilancia y de la represión de modo directo. A pesar de que la vigilancia se volvía algo cotidiano, más o menos naturalizada, no dejaba de tener efectos sobre las experiencias de los estudiantes.

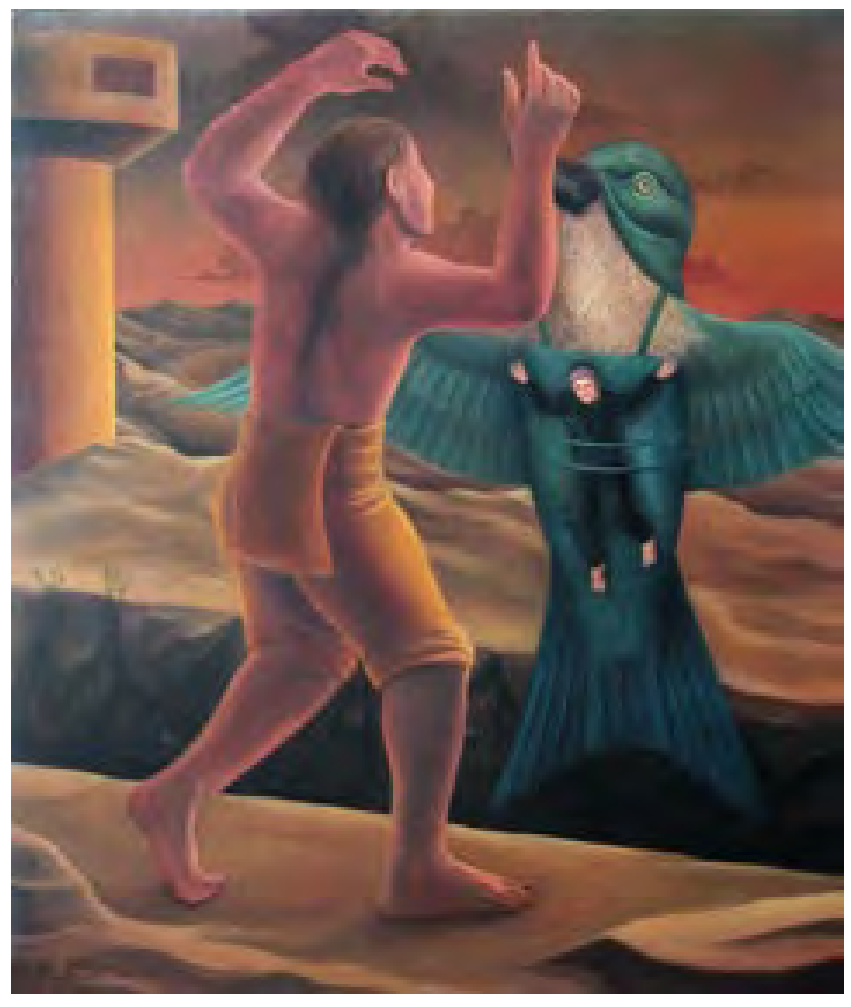

Pablo Serrano, Aprendiz de pájaro, oleo/tela, $50 \times 40 \mathrm{~cm}$, colección particular, 2006. 


\section{A modo de cierre}

A lo largo del presente artículo observamos cómo las experiencias respecto de la represión y el disciplinamiento de los y las estudiantes se vieron influenciadas por múltiples factores. La edad y el momento de ingreso a la universidad, su militancia y la facultad donde estudiaron son elementos que tenemos que considerar para evitar caer en generalizaciones simplistas que pierdan de vista que los dispositivos represivos tuvieron peculiaridades en su aplicación en cada facultad. En este punto debemos problematizar las afirmaciones generales de Pedrosa (2001) acerca de que la política represiva y universitaria de la dictadura se aplicó sin matices en todas las universidades.

También, hemos dado cuenta de que hubo momentos con características particulares en función del contexto político y social nacional que marcaron etapas diferenciadas en la aplicación de la política represiva y de disciplinamiento, así como también condicionaron las trayectorias del estudiantado. De este modo, sostenemos que estas variables que aportan elementos para comprender la relativa heterogeneidad de representaciones y experiencias entre quienes estudiaron durante la etapa y también las operaciones de naturalización y acomodamiento a la realidad vigente.

Hemos observado cómo los estudiantes de la UBA aprendieron a estudiar y pasar sus días de juventud en los claustros en un contexto represivo. Los mismos desarrollaron estrategias para convivir con la vigilancia y controles permanentes, generando un cierto proceso de acostumbramiento y naturalización de la situación. Dicha aceptación o normalización de ningún modo supuso actitudes de acuerdo y apoyo total hacia el régimen por parte de los sectores estudiantiles. Por un lado, los y las jóvenes desarrollaron estrategias de resguardo de su seguridad, de vigilancia y control entre pares, de retirada de la vida política pública. A la vez, los aprendizajes de las y los estudiantes posibilitaron que desplegaran estrategias de cuidado y compañerismo entre sí, potenciando el surgimiento de nuevas solidaridades, complicidades y vínculos con sus compañeros. Por otro lado, construyeron diversos tipos de vínculos con los representantes de las fuerzas represivas y tipo de actitudes hacia su presencia.
Debemos destacar, en ese sentido, las recurrentes menciones a que la actividad de las mismas en los claustros era parte constitutiva de la realidad cotidiana y que paradójicamente no son recordadas como un peligro o amenaza real, a diferencia de soldados y policías en la vía pública. Incluso, aún con su presencia, gracias a los vínculos cotidianos establecidos entre estudiantes y policías, fue factible ingresar materiales prohibidos a las facultades e ir recuperando cierta actividad estudiantil. En este sentido, debe quedar planteado que la aplicación de la política represiva y de disciplinamiento en los claustros no logró desaparecer de manera absoluta la actividad estudiantil (cultural, recreativa, política) como tampoco obtuvo consenso generalizado entre el estudiantado (Seia, 2016a y b; Seia, 2017; Perel et al, 2006; Pedrosa, 2001). 


\section{Notas}

1. En este artículo retomamos las ideas desarrolladas en nuestra tesis de maestría La Universidad de Buenos Aires (UBA) entre la "Misión Ivanissevich" y la última dictadura (1974-1983). Represión, "reordenamiento" y reconfiguraciones de la vida estudiantil (2016).

2. Se conoce que hubo 130 desaparecidos vinculados a la Facultad de Arquitectura, 27 de la carrera de Psicología, 72 de la Facultad de Ciencias Económicas, 70 de la Facultad de Ciencias Exactas y Naturales; 61 en Ingeniería y 127 en Filosofía y Letras.

3. Los rectores de la UBA fueron: Alberto Constantini (agosto 1976-septiembre 1976), Libertario Rabasa (septiembre 1976-febero 1977), Luis Cabral (febrero 1977-agosto 1978), Alberto Donnes (agosto 1978-noviembre 1978), Lucas Lennon (noviembre 1978-noviembre 1981), Alberto Rodríguez Varela (diciembre 1981-diciembre 1982) y Carlos Segovia Fernández (diciembre 1982- diciembre 1983).

\section{Referencias}

\section{Fuentes}

Consejo de Rectores de Universidades Nacionales, Asamblea (1982). "Exposición de la Asesoría de Comunicación Social acerca del accionar de la oposición política y del oponente subversivo sobre el sector estratégico educativo". Bs. As.

FFAA (1979). "El Terrorismo en Argentina”. Bs. As.

Fuerza Aérea Argentina (1982). "Informe periódico de la Delegación Militar de Fuerza Aérea. Reunión de rectores de universidades nacionales, subsecretario de educación, asesor de comunicación social y delegados militares. Tema: neutralización del activismo estudiantil." Bs. As.

\section{Testimonios}

Entrevistas a Claudio Spiguel (2013), Carlos Astarita (s/d), Alberto Lettieri (s/d) y Magdalena Frere (2013), realizadas por el Programa "Universidad y Dictadura" de la Cátedra Libre de DD.HH. de la Facultad de Filosofía y Letras.

Entrevistas a Irina Izaguirre (2012), Haydée Pizarro (2012), Ricardo Durán (2014), Juan Carlos Pedraza (2016), Arturo Romano (2013), Gabriel Bilmes (2011), Juan Pablo Paz (2007) y Sara Aldabe (2010) realizadas por Gabriel Rocca de la FCEN para la sección "Universidad y Dictadura" del portal NexCiencia, de la Facultad de Ciencias Exactas y Naturales.
4. Perel et al. (2009) describen que los estudiantes llamaban a los policías de civil por la película "Sérpico" (1973) donde Al Pacino interpreta el papel de un policía encubierto.

5. De los 124 desaparecidos y/o asesinados en Filosofía y Letras, por ejemplo, 38 cayeron durante 1976, 43 en 1977, 10 en 1978 y 3 en 1979 (Cátedra Abierta de DDHH). Durante 1976 y 1977 se concentró el mayor número de víctimas del accionar represivo; en los años posteriores esa cifra disminuyó. Hacia mediados de 1978, no sólo las organizaciones guerrilleras, sino también los partidos de izquierda, los delegados sindicales y las agrupaciones estudiantiles habían sido diezmados (Novaro y Palermo, 2010).
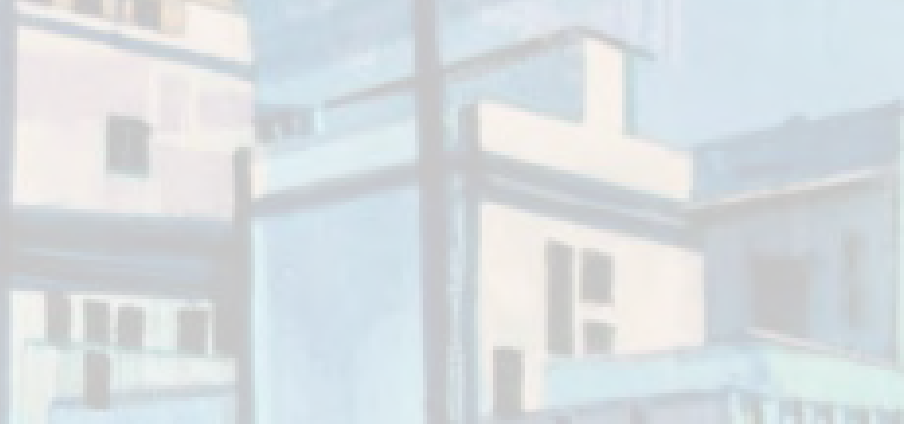

Fuerza Aérea Argentina, Jefatura II de Inteligencia (1982). "Comunicación Escrita Interna $\mathrm{N}^{\circ} 20659$, e informe de inteligencia”. Bs. As.

PEN (1976a). Ley 21.276 "Prioridad para la normalización de las universidades nacionales".

PEN (1976b). "Bases para la intervención de las fuerzas armadas en el proceso nacional".

UBA, Rectorado. Resoluciones Rectorales, años 1974-1983.

Entrevistas a Sonia, Nancy, Jorge Cernadas, Eduardo, Lucas Luchilo, Alejandro Cattaruzza, Daniel Sierra, Patricio Geli, Pablo Alabarces, Patricia Berrotarán, Marcelo (Filosofía y Letras); Gabriel Bilmes, Juan Pablo Paz, Carolina Vera, Pablo Mauas, Virginia Ferraro (Ciencias Exactas); María Giurlanda (Ingeniería); Sara Sánchez (Medicina); Víctor Cipolla, Mariano Ciaffardini, Jorge Kreyness, Carlos Cebey (Derecho): Daniel Lazewiki, Andrés Delich (Ciencias Económicas); Luis García Fanlo, Andrés Delich (Sociología), realizada por G. Seia durante 2015. Buenos Aires. 


\section{Bibliografía}

Águila, G. (2014). La Universidad Nacional de Rosario en dictadura (1976-1983): depuración, "normalización" y reestructuración institucional. PolHis (núm. 14).

Algañaraz Soria, V. (2014). Reestructuración universitaria en clave autoritaria: política y accionar de los rectores de la Universidad Nacional de San Juan durante la última dictadura militar (19761983). PolHis, Revista del Programa Interuniversitario de Historia Política. (Núm.14).

Bonavena, P. (2008). El movimiento estudiantil universitario frente a la Misión Ivanissevich: el caso de la Universidad de Buenos Aires. En IV Jornadas de Trabajo sobre Historia reciente, Rosario.

Bonavena, P. (2016). El Movimiento estudiantil de la Facultad de Arquitectura y Urbanismo de la UBA: del golpe de Onganía al Arquitecturazo. En VI Jornadas de Estudio sobre el Movimiento Estudiantil, Buenos Aires.

Buchbinder, Pablo (2010). Historia de las universidades argentinas. Buenos Aires: Sudamericana.

Califa, J. (2015). A los golpes con el golpe. El movimiento estudiantil frente a la intervención de la Universidad de Buenos Aires, 1966. Conflicto Social, Revista del Programa de Investigaciones sobre Conflicto Social, vol. 8.

Califa, J. y Millán M. (2016). El movimiento estudiantil como objetivo de la represión. Un estudio sobre el caso de la UBA entre 1966 y 1976. PolHis Revista Bibliográfica del Programa Interuniversitario de Historia Política, vol. 8.

De Riz, L. (2000). La política en suspenso 1966 - 1976, Buenos Aires: Paidós.

Díaz de Guijarro, E. y otros. (2015). Historia de la Facultad de Ciencias Exactas y Naturales. UBA. Buenos Aires: EUDEBA.

Franco, M. (2012). Un enemigo para la nación: orden interno, violencia $y$ «subversión, 1973-1976. Buenos Aires: Fondo de Cultura Económica.

Friedemann, S. (2015). La Universidad Nacional y Popular de Buenos Aires (1973-1974) Una reforma universitaria inconclusa, Tesis doctoral en Ciencias Sociales, Universidad de Buenos Aires, Buenos Aires, inédita.

Gil, G. y Díaz M. (2014). Continuidades, “orden” y “despolitización”. La Universidad Nacional de Mar del Plata en los años de dictadura (1976-1983). PolHis. (Núm. 14).

Izaguirre, I. (2011). La Universidad y el Estado terrorista. La Misión Ivanissevich. Conflicto Social, (núm. 5).

Kaufamnn, C. (dir.) (2001 y 2003). Dictadura y Educación. Tomo 1 y 2. Buenos Aires: Miño y Dávila.

Luciani, L. (2017). Juventud en dictadura: representaciones, políticas y experiencias juveniles en Rosario: 1976-1983. La Plata: Universidad Nacional de La Plata; Posadas: Universidad Nacional de Misiones; Los Polvorines: Universidad Nacional de General Sarmiento.

Mendonça, M. (2016). Entre botas y votos. Las políticas universitarias durante la "Revolución Argentina". Del golpe de Estado de 1966 a las elecciones de 1973 (tesis doctoral), Buenos Aires, IDES-UNGS.

Millán, M. (2015). Conflicto universitario y estudiantil en la UBA durante el rectorado de Rodolfo Puiggrós (junio - octubre de 1973). Conflicto Social (IIGG/UBA), vol. 8 (núm. 14).

Novaro, M. y Palermo V. (2003). La dictadura militar. (1976-1983). Del golpe de Estado a la restauración democrática. Buenos Aires: Paidós.
Orbe, P. (2014). El "proceso de reorganización" de los claustros: el impacto político-educativo de la última dictadura en la Universidad Nacional del Sur. PolHis, (núm. 14).

Pedrosa, F. (2002). La universidad y los estudiantes frente a la dictadura militar. En R. Marsiske. (Coord.), Movimientos estudiantiles en la historia de América Latina. México: UNAM.

Perel, P., Raíces E. y Perel M. (2006). Universidad y dictadura. Derecho, entre la liberación y el orden (1973-1983). Buenos Aires: Ediciones del CCC.

Pis Diez, N. (2016). "Es hora de jugar la Universidad". Una reconstrucción de las luchas reformistas en las calles platenses durante la "Laica o Libre" (septiembre - octubre de 1958). Conflicto Social, Revista del Programa de Investigaciones sobre Conflicto Social, vol. 9 (núm. 15).

Polak, L. y Gorbier J. (1994). El movimiento estudiantil Argentino (Franja Morada 1976-1986). Buenos Aires: CEAL.

Rodríguez, L. (2014). La Universidad Nacional de La Plata entre 1973 y 1983. PolHis. (Núm 14).

Rodríguez, L. (2015). Universidad, peronismo y dictadura. 1973-1983. Buenos Aires: Prometeo.

Seia, G. (2014). Rectores de la Universidad de Buenos Aires entre 1974 y 1983: curricula vitae, discursos y comunicados de prensa. Cuaderno ${ }^{\circ} 5$.

Seia, G. (2015a). Reconfiguraciones de la vida estudiantil durante la última dictadura. Apuntes sobre los casos de las Facultades de Filosofía y Letras y Ciencias Exactas y Naturales de la Universidad de Buenos Aires (1976-1981). RBBA. Revista Binacional BrasilArgentina, vol. 4, (núm. 1).

Seia, G. (2016a). La Universidad de Buenos Aires (UBA) entre la "Misión Ivanissevich" y la última dictadura (1974-1983). Represión, "reordenamiento" y reconfiguraciones de la vida estudiantil (tesis de maestría en Historia Contemporánea). IDH-UNGS, Los Polvorines.

Seia, G. (2016b). Militancia, oposición y resistencia estudiantil en la Universidad de Buenos Aires durante la etapa final de la última dictadura (1981-1983). Historia, voces y memoria, (núm. 10).

Seia, G. (2016c). La Política Universitaria contra el "Problema Universitario" durante la última dictadura. Un estudio de caso sobre la Universidad de Buenos Aires entre 1976 y 1980". En IX Jornadas de Sociología, Universidad Nacional de La Plata, La Plata.

Seia, G. (2017). Las Revistas Estudiantiles en la Universidad de Buenos Aires durante la última dictadura (1976-1983). Un estudio exploratorio sobre las prácticas culturales del estudiantado universitario en la Facultad de Ciencias Exactas y Naturales. En I Coloquio de Historia de las Juventudes, La Condición Juvenil en América Latina, Culturas, Identidades y Movimientos Estudiantiles, IISUE-UNAM, México.

Vera de Flachs, C. (2015). Escarceos estudiantiles en época de dictadura, Argentina. En R. Marsiske. (Coord.), Movimientos estudiantiles en la historia de América Latina. México: IISUE-UNAM. 